\title{
Biosynthesis of phlorisovalerophenone and 4-hydroxy-6-isobutyl-2-pyrone in Escherichia coli from glucose
}

Wei Zhou ${ }^{1,2,3}$, Yibin Zhuang ${ }^{1,2}$, Yanfen Bai ${ }^{1,2,3}$, Huiping Bi ${ }^{1,2^{*}}$, Tao Liu ${ }^{1,2^{*}}$ and Yanhe Ma ${ }^{1}$

\begin{abstract}
Background: Type III polyketide synthases (PKSs) contribute to the synthesis of many economically important natural products, which are typically produced by direct extraction from plants or synthesized chemically. For example, humulone and lupulone (Fig. 1a) in hops (Humulus lupulus) account for the characteristic bitter taste of beer and display multiple pharmacological effects. 4-Hydroxy-6-methyl-2-pyrone is a precursor of parasorboside contributing to insect and disease resistance of plant Gerbera hybrida, and was recently demonstrated to be a potential platform chemical.
\end{abstract}

Results: In this study, we achieved simultaneous biosynthesis of phlorisovalerophenone, a key intermediate of humulone biosynthesis and 4-hydroxy-6-isobutyl-2-pyrone in Escherichia coli from glucose. First, we constructed a biosynthetic pathway of isovaleryl-CoA via hydroxy-3-methylglutaryl CoA followed by dehydration, decarboxylation and reduction in E. coli. Subsequently, the type III PKSs valerophenone synthase or chalcone synthase from plants were introduced into the above E. coli strain, to produce phlorisovalerophenone and 4-hydroxy-6-isobutyl-2-pyrone at the highest titers of 6.4 or $66.5 \mathrm{mg} / \mathrm{L}$, respectively.

Conclusions: The report of biosynthesis of phlorisovalerophenone and 4-hydroxy-6-isobutyl-2-pyrone in E. coli adds a new example to the list of valuable compounds synthesized in E. coli from renewable carbon resources by type III PKSs.

Keywords: Isovaleryl-CoA, Phlorisovalerophenone, 4-Hydroxy-6-isobutyl-2-pyrone, Valerophenone synthase, Escherichia coli

\section{Background}

A large number of natural products are synthesized by type III polyketide synthases (PKSs). These compounds play an important role in human nutrition and health, and have recently expanded their roles as platform chemicals. Acylphloroglucinol derivatives have been isolated from a number of plants [1-3]. Their chemical structures and intriguing biological activities have attracted increasing attention in recent years. Humulone and lupulone (Fig. 1a) in hops (Humulus lupulus) account for the characteristic bitter taste of beer and display multiple

\footnotetext{
*Correspondence: bi_hp@tib.cas.cn; liu_t@tib.cas.cn

${ }^{1}$ Tianjin Institute of Industrial Biotechnology, Chinese Academy

of Sciences, Tianjin 300308, China

Full list of author information is available at the end of the article
}

pharmacological effects [4]. Hyperforin (Fig. 1a) is one of the main active constituents in extracts of Hypericum perforatum used for the treatment of depression [5]. The 4-hydroxy-2-pyrone analogues display multiple biological activities and are valuable pharmaceutical precursors [6]. 4-Hydroxy-6-methyl-2-pyrone, also known as triacetic acid lactone (TAL, Fig. 1b) is a precursor of gerberin and parasorboside, which could protect Gerbera hybrid from fungal pathogens and attack by insects [7]. Fistupyrone (4-hydroxy-6-isovaleryl-2-pyrone) (Fig. 1b) was isolated from Streptomyces sp. TP-A0569 and showed inhibition of the infection of Chinese cabbage by a fungus Alternaria brassicicola [8]. Germicidin homologs (Fig. 1b) arisen from streptomyces inhibit spore germination and $\mathrm{Na}^{+} / \mathrm{K}^{+}$ATPase $[9,10]$. More recently, 
a<smiles>Oc1cc(O)cc(O)c1</smiles>

phloroglucinol<smiles>C/C=C/C[C@]1(CC=C(C)C)C(=O)C(C(=O)CC(C)C)=C(O)C(CC=C(C)C)=C1O</smiles>

humulone

b<smiles>Cc1cc(O)cc(=O)o1</smiles><smiles>CC(C)C(=O)c1c(O)cc(O)cc1O</smiles>

PIBP<smiles>CC(C)=CCC1=C(O)C(O)(CCC(C)C)C(O)C(C(=O)CC(C)C)=C1O</smiles>

lupulone<smiles>CC(C)c1cc(O)cc(=O)o1</smiles>

HIPP<smiles>CC(C)CC(=O)c1c(O)cc(O)cc1O</smiles>

PIVP
TAL<smiles>CC(C)CCc1cc(O)cc(=O)o1</smiles>

fistupyrone<smiles>CCc1c(O)cc(C(C)C)oc1=O</smiles>

germicidin $\mathrm{A}$

Fig. 1 Examples of phloroglucinols (a) and 2-pyrones (b) synthesized by type III PKS. PIBP phlorisobutyrophenone; PIVP phlorisovalerophenone; TAL 4-hydroxy-6-methyl-2-pyrone (triacetic acid lactone); HIPP 4-hydroxy-6-isopropyl-2-pyrone; HIBP 4-hydroxy-6-isobutyl-2-pyrone

4-hydroxy-2-pyrones were reported to be potential platform chemicals (Additional file 1: Figure S1a) [11-13].

Advances in synthetic biology and metabolic engineering greatly promoted biosynthesis of a variety of valuable compounds, for instance, flavonoids produced by type III PKSs [14, 15] in microbial hosts. Biological production of phloroglucinol (Fig. 1a) and TAL (Fig. 1b) from glucose has also been accomplished using genetically modified microorganisms [6, 16-19]. However, biosynthesis of acylphloroglucinols and TAL analogues from renewable feedstocks in Escherichia coli has not been well investigated.
The acylphloroglucinol cores, phlorisovalerophenone (PIVP) and phlorisobutyrophenone (PIBP) are formed by condensation of three malonyl-CoA-derived acetate units with isovaleryl-CoA or isobutyryl-CoA as the starter units $[4,20]$. The enzyme valerophenone synthase (VPS) involved in the formation of PIVP in the biosynthesis of humulone has been characterized [21-23]. VPS is a homologue of chalcone synthase (CHS). They share the same reaction mechanism namely claisen condensation, but differ in substrate specificity. CHS catalyzes condensation of one molecule of $p$-coumaroyl-CoA and three molecules of malonyl-CoA to form naringenin-chalcone. 
However, VPS preferentially uses isovaleryl-CoA or isobutyryl-CoA instead of $p$-coumaroyl-CoA as the starter unit to condense with three molecules of malonyl-CoA to form PIVP [21, 23]. Recently, dual functional CHS/VPS have also been reported. A CHS, FvCHS2-1 from strawberry (Fragaria vesca) was identified to be responsible for acylphloroglucinols synthesis in strawberry fruit [3]. Intriguingly, in vitro experiments demonstrated that type III PKSs such as VPS and CHS usually also synthesize TAL analogues, 4-hydroxy-6-isobutyl2-pyrone (HIBP) and 4-hydroxy-6-isopropyl-2-pyrone (HIPP) (Fig. 1b), carrying isobutyl or isopropyl at position 6 with isovaleryl-CoA or isobutyl-CoA as the starter unit and malonyl-CoA as the extender unit.

In this work, we engineered the synthesis of isovalerylCoA in E. coli by recruiting a biosynthetic pathway via hydroxy-3-methylglutaryl CoA (HMG-CoA), an intermediate of the mevalonate pathway (Fig. 2a) [24]. The VPS from hops (Humulus lupulus) HIVPS, the CHS from strawberry (Fragaria vesca) FvCHS2-1 and a newly cloned $\mathrm{CHS}$ from $H$. perforatum $\mathrm{HpCHS}$ were introduced into the isovaleryl-CoA producing $E$. coli strain separately, led to the simultaneous production of PIVP and HIBP at different ratios. The highest titers of PIVP and HIBP produced by the recombinant strains reached 6.4 and $66.5 \mathrm{mg} / \mathrm{L}$, respectively. This work adds new products to the list of valuable compounds biosynthesized in E. coli using type III PKSs and lays a foundation for microbial synthesis of not only acylphloroglucinol derivatives, but also potential platform chemicals.

\section{Methods}

\section{Strains, plasmids and medium}

Strains and plasmids used in this study are listed in Table 1. Luria-Bertani (LB) medium was used for the propagation of $E$. coli cells for plasmid construction. Terrific Broth (TB) medium (12 g/L tryptone, $24 \mathrm{~g} / \mathrm{L}$ yeast extract, $4 \mathrm{~mL} / \mathrm{L}$ glycerol, $2.3 \mathrm{~g} / \mathrm{L} \mathrm{KH}_{2} \mathrm{PO}_{4}$, and $12.5 \mathrm{~g} / \mathrm{L}$ $\left.\mathrm{K}_{2} \mathrm{HPO}_{4}\right)$ and modified M9 medium $(1 \times$ M9 minimal salts, $20 \mathrm{~g} / \mathrm{L}$ glucose, $10 \mathrm{~g} / \mathrm{L}$ yeast extract, $2 \mathrm{mM} \mathrm{MgSO}_{4}$, and $0.1 \mathrm{mM} \mathrm{CaCl}_{2}$ ) were used for protein expression and PIVP production, respectively. Ampicillin $(100 \mathrm{mg} / \mathrm{L})$ and streptomycin $(100 \mathrm{mg} / \mathrm{L})$ were added to the medium as needed.

\section{Construction of plasmids}

Oligonucleotide primers used in this study are summarized in Additional file 1: Table S1. AibA, aibB, $a i b C$ and liuC were PCR-amplified from Myxococcus xanthus (CGMCC 1.3865) genome with primer pairs aibA-F/ aibA-R, aibB-F/aibB-R, aibC-F/aibC-R or liuC-F/liuC-R, respectively. The amplified gene products of $a i b A, a i b B$ and $a i b C$ were digested with restriction enzyme and simultaneously subcloned into the multiple cloning sites (MCS) 1 of pETDuet-1 by Golden Gate [25] cloning using Aarl. Subsequently, the gene product of liuC was digested and ligated into the above resulting plasmid using HindIII/AflII to yield the final plasmid pETA. Here, the sequences of T7 promoter, lac operator and ribosome binding site (RBS) from pETDuet-1 were incorporated into the primer design, so each gene was driven by a single T7 promoter. ERG10 and ERG13 were PCR-amplified from Saccharomyces cerevisiae BY4742 genome with primer pairs ERG10-F/ERG10-R or ERG13F/ERG13-R, respectively. First, the ERG13 fragment was digested and cloned into $N c o I / B a m H I$ sites of pCDFDuet-1. The ERG10 fragment was digested and ligated into the above plasmid via $\mathrm{NdeI} / \mathrm{XhoI}$ sites, generating the recombinant plasmid pCDF-E. The HlVPS gene from hops (GenBank: AB015430.1) was codon optimized for expression in E. coli and synthesized by Shanghai Generay Biotech Co., Ltd. (Shanghai, China), designated as $H l V P S^{s y n}$ (Additional file 1: Table S2). HlVPS $S^{\text {syn }}$ was PCR-amplified with primer pair VPS-F/VPS-R, and cloned into pET28a through NdeI/AflII sites generating the intermediate expression vector pET28a-HIVPS. The T7-HlVPS ${ }^{\text {syn }}$ fragment with a T7 promoter and an $\mathrm{N}$-terminal His tag was PCR-amplified from pET28aHIVPS with primer pair T7VPS-F/T7VPS-R, digested and ligated into pCDF-E via NotI/AflII to generate the final recombinant plasmid pCDF-EV1. FvCHS2-1 from strawberry (GenBank: XM_004306495.1) was codon optimized for expression in E. coli and synthesized by Shanghai Generay Biotech Co., Ltd. (Shanghai, China), designated as $\mathrm{FvCHS}_{2} \mathrm{I}^{\text {syn }}$ (Additional file 1: Table S2). $H p C H S$ was obtained from the mRNA of $H$. perforatum by RT-PCR. RNA of $H$. perforatum callus was isolated by RNAprep pure Plant Kit from Tiangen Biotech Co., Ltd. (Beijing, China). Reverse transcription was performed using the First Strand cDNA Synthesis Kit from Toyobo Co., Ltd. (Osaka, Japan). PCR reactions were performed using High Fidelity PCR system (Fermentas, Germany) with primer pair HpCHS-F/HpCHS-R. The HpCHS sequence was submitted into NCBI database (GenBank: KU180217). $F v C H S 2-1^{s y n}$ and $H p C H S$ were ligated into pCDF-E individually using the same method as pCDFEV1 construction, yielding pCDF-EV2 and pCDF-EV3, respectively.

\section{Biosynthesis of isovaleryl-CoA in E. coli}

The plasmids, pET-A and pCDF-E, were electroporated into BL21 (DE3), generating the recombinant strain APG-IV. The strain APG-0 harboring pETDuet-1 and pCDFDuet-1 was used as the control. A $1 \mathrm{~mL}$ aliquot of the overnight cultured single colony was inoculated into $50 \mathrm{~mL} \mathrm{LB}$ medium containing antibiotics and 


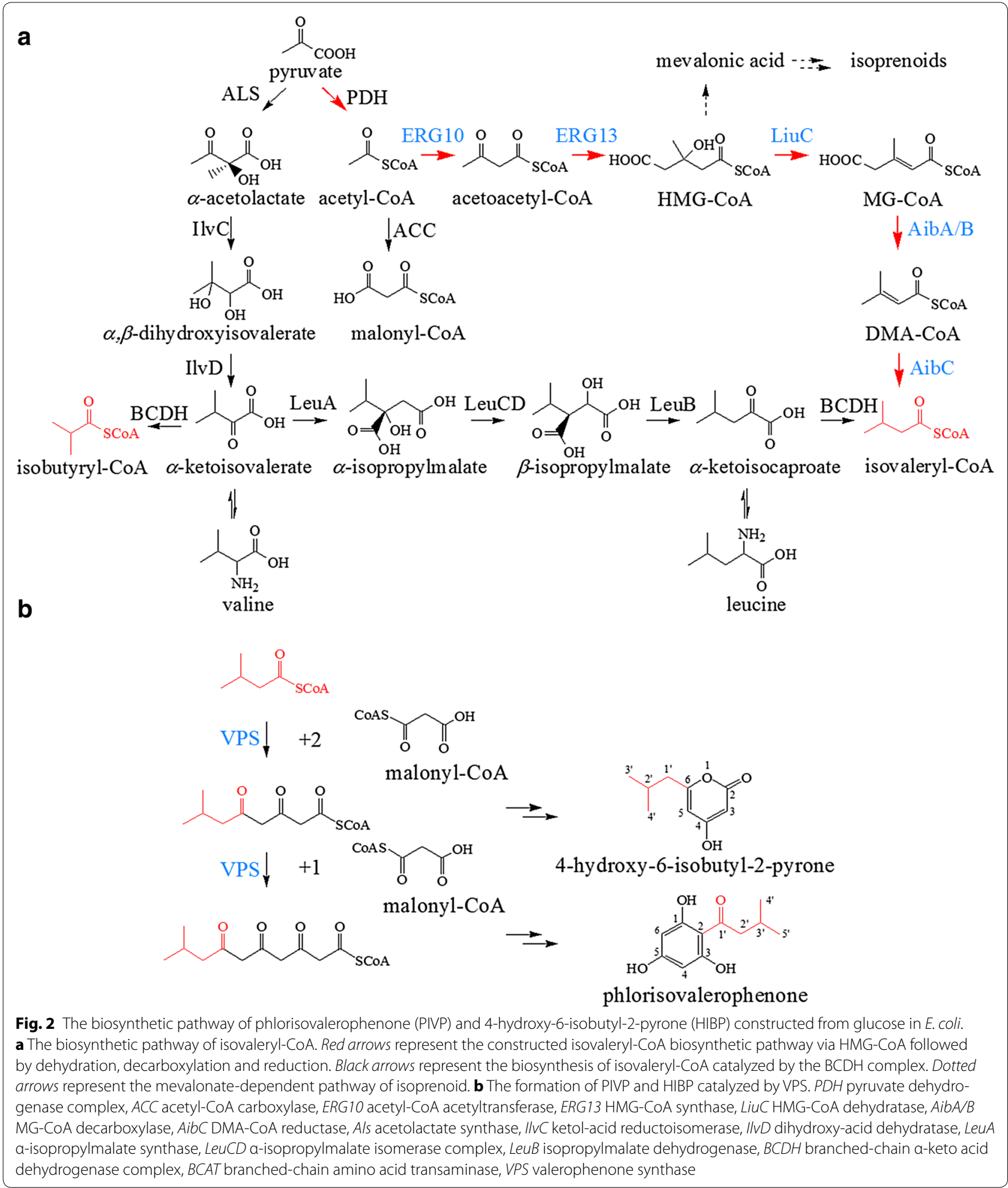

cultivated at $37{ }^{\circ} \mathrm{C}, 200 \mathrm{rpm}$ with shaking. When $\mathrm{OD}_{600}$ of the culture reached $0.6,0.1 \mathrm{mM}$ isopropyl- $\beta-\mathrm{D}$ thiogalactoside (IPTG) was added to induce recombinant protein expression at $30{ }^{\circ} \mathrm{C}$ for $12 \mathrm{~h}$. Subsequently, the cells were centrifuged $\left(4000 \mathrm{rpm}, 30{ }^{\circ} \mathrm{C}, 10 \mathrm{~min}\right)$, washed and resuspended in $50 \mathrm{~mL}$ modified M9 medium and 
Table 1 Bacterial strains and plasmids

\begin{tabular}{|c|c|c|}
\hline Name & Description & Reference \\
\hline \multicolumn{3}{|l|}{ Plasmids } \\
\hline pETDuet-1 & pBR322 ori with $\mathrm{P}_{\mathrm{T} 7} ; \mathrm{Amp}^{\mathrm{R}}$ & Novagen \\
\hline pCDFDuet-1 & CDF ori with $\mathrm{P}_{T 7} ; \mathrm{Sm}^{\mathrm{R}}$ & Novagen \\
\hline pET-A & pETDuet-1 carrying $a i b A, a i b B$, aibC and liuC & This study \\
\hline pCDF-E & pCDFDuet- 1 carrying ERG10 and ERG13 & This study \\
\hline pCDF-EV1 & $\begin{array}{l}\text { PCDFDuet-1 carrying ERG10, ERG13 and } \\
\text { HIVPS Syn }\end{array}$ & This study \\
\hline pCDF-EV2 & $\begin{array}{l}\text { pCDFDuet-1 carrying ERG10, ERG13 and } \\
\text { FvCHS2-1 } 7^{\text {syn }}\end{array}$ & This study \\
\hline pCDF-EV3 & $\begin{array}{l}\text { pCDFDuet-1 carrying ERG10, ERG13 and } \\
\text { HpCHS }\end{array}$ & This study \\
\hline \multicolumn{3}{|l|}{ Strains } \\
\hline $\begin{array}{l}\text { Myxobac- } \\
\text { teria }\end{array}$ & Myxococcus xanthus (CGMCC 1.3865) & CGMCC \\
\hline Yeast & Saccharomyces cerevisiae BY4742 & 29 \\
\hline APG-0 & $\begin{array}{l}\text { E. coli BL21 (DE3) with pETDuet-1 and } \\
\text { pCDFDuet-1 }\end{array}$ & This study \\
\hline APG-IV & E. coli BL21 (DE3) with pET-A and pCDF-E & This study \\
\hline APG-1 & E. coli BL21 (DE3) with pET-A and pCDF-EV1 & This study \\
\hline APG-2 & E. coli BL21 (DE3) with pET-A and pCDF-EV2 & This study \\
\hline APG-3 & E. coli BL21 (DE3) with pET-A and pCDF-EV3 & This study \\
\hline
\end{tabular}

CGMCC China general microbiological culture collection center

cultured at $30{ }^{\circ} \mathrm{C}$ for $3 \mathrm{~h}$. The cell culture was chilled on ice for $10 \mathrm{~min}$. Cells were harvested by centrifugation (4000 rpm, $4{ }^{\circ} \mathrm{C}, 10 \mathrm{~min}$ ) and resuspended in $1.5 \mathrm{~mL}$ extraction buffer (methanol:acetonitrile:water $=2: 2: 1$, $\mathrm{v} / \mathrm{v}$, with $0.1 \%$ formic acid). After sonication and centrifugation, $50 \mu \mathrm{L}$ of the supernatants were used for HPLC and LC-MS analysis.

\section{Biosynthesis of PIVP and HIBP in E. coli}

Escherichia coli strain BL21 (DE3) was transformed with plasmids pET-A and pCDF-EV1, pET-A and pCDF-EV2 or pET-A and $\mathrm{pCDF}-\mathrm{EV} 3$ to generate the recombinant strains APG-1, APG-2 or APG-3, respectively. Two stage fermentation processes were applied to achieve the production of PIVP and HIBP. The first stage is for pathway genes overexpression, and recombinant strains were cultivated in TB medium. Single colonies were cultured in $3 \mathrm{~mL} \mathrm{LB}$ medium with antibiotics at $37^{\circ} \mathrm{C}$ for $7 \mathrm{~h}$ as seed culture, $1 \mathrm{~mL}$ of which was transferred into $50 \mathrm{~mL}$ TB medium with antibiotics and cultured in a shake flask at $37{ }^{\circ} \mathrm{C}$ until an $\mathrm{OD}_{600}$ of 1.2 followed by addition of $0.1 \mathrm{mM}$ IPTG. To determine optimal temperature for fermentation conditions at the stage, the culture was incubated at 16,23 or $30{ }^{\circ} \mathrm{C}$ for $16 \mathrm{~h}$. Different concentrations of IPTG $(0.01,0.05,0.1,0.2$ or $0.3 \mathrm{mM})$ were also added in the TB medium to determine the optimal concentrations. The cell pellets were harvested by centrifugation and resuspended in an equal volume of modified M9 medium and fermented at $30{ }^{\circ} \mathrm{C}, 200 \mathrm{rpm}$ for secondary metabolite production as the second stage. Time course for production of PIVP and HIBP by strain APG-1 was determined for 12, 24, 36, 48 and $60 \mathrm{~h}$. Subsequently, the culture broth was centrifuged at $4000 \mathrm{rpm}$ for $10 \mathrm{~min}$. The supernatants were extracted with an equal volume of ethyl acetate twice, concentrated by rotary evaporator, and re-dissolved in $1.5 \mathrm{~mL}$ methanol. To extract the intracellular metabolites, cell pellets were resuspended in $10 \mathrm{~mL} 80 \%$ acetone, sonicated, centrifuged and the supernatant was evaporated to remove the acetone. The procedure was repeated once. Subsequently, the remaining water phase was also extracted with an equal volume of ethyl acetate twice and re-dissolved in $1.5 \mathrm{~mL}$ methanol as the supernatant of the fermented broth. Then $20 \mu \mathrm{L}$ extraction samples of the fermentation broth or the cell pellets were analyzed by HPLC-MS separately.

\section{Compounds purification}

$2 \mathrm{~L}$ fermentation broth of the recombinant strain APG-1 was centrifuged to separate into supernatant and cell pellets. After crude extraction using the above protocol, purification of HIBP and PIVP was conducted by semipreparative HPLC performed on a Shimadzu LC-6 AD with SPD-20A detector. The HPLC conditions were as follows: solvent $\mathrm{A}=\mathrm{H}_{2} \mathrm{O}$; solvent $\mathrm{B}=$ methanol; flow rate: $4 \mathrm{~mL} / \mathrm{min}, 0-5 \min 90 \% \mathrm{~A}$ and $10 \% \mathrm{~B}, 6-40 \mathrm{~min}$ $90 \% \mathrm{~A}$ and $10-100 \% \mathrm{~B}$ (linear gradient), 41-45 min $100 \%$ B. A YMC-pack ODS-A $(10 \times 250$ mm; particle size, $5 \mu \mathrm{m}$ ) was used as the stationary phase.

\section{Chemical analysis and quantification}

HPLC-MS was performed on an Agilent 1260 system with 1260 Infinity UV detector and a Bruker microQTOF II mass spectrometer equipped with an ESI ionization probe. The innoval C18 column $(4.6 \times 250 \mathrm{~mm}$; $5 \mu \mathrm{m}$ particle size) was used in this study. The HPLC conditions for isovaleryl-CoA, HIBP and PIVP were as follows: solvent $\mathrm{A}=\mathrm{H}_{2} \mathrm{O}$ (containing $20 \mathrm{mM}$ ammonium acetate for the analysis of isovaleryl-CoA, or containing $0.1 \%$ formic acid for the analysis of HIBP and PIVP); solvent $\mathrm{B}=$ methanol; flow rate $=1 \mathrm{~mL} / \mathrm{min} ; 0-5 \mathrm{~min}$ $95 \% \mathrm{~A}$ and $5 \% \mathrm{~B}, 6-45 \min 95 \% \mathrm{~A}$ and $5 \% \mathrm{~B}$ to $100 \%$ B (linear gradient). All these products were detected at $254 \mathrm{~nm}$. Standard calibration curve of isovaleryl-CoA was generated with a series of known concentrations of the isovaleryl-CoA standard purchased from SigmaAldrich (Milwaukee, WI, USA). Standard calibration curves of HIBP and PIVP were generated with a series of known concentrations of the purified compounds from large scale fermentation. All experiments were carried 
out in triplicate and repeated at least twice. The titer was presented as mean $\pm \mathrm{SD}$.

\section{NMR analysis}

NMR experiments were performed on a Bruker Avance 400 (Karlsruhe, Germany). The sample were dried by evaporation and dissolved in $500 \mu \mathrm{L}$ of DMSO- $d_{6}$ and transferred into $2.5 \mathrm{~mm}$ NMR tube. Chemical shifts were expressed in $\delta(\mathrm{ppm})$ and coupling constants $(J)$ were given in $\mathrm{Hertz}(\mathrm{Hz})$ (Additional file 1: Figure S2).

$H I B P{ }^{1} \mathrm{H}-\mathrm{NMR}$ (DMSO- $\left.d_{6}, 400 \mathrm{MHz}\right) \delta 5.94(\mathrm{~d}$, $J=2.1 \mathrm{~Hz}, 1 \mathrm{H}), 5.21(\mathrm{~d}, J=2.1 \mathrm{~Hz}, 1 \mathrm{H}), 2.30(\mathrm{~d}$, $J=7.2 \mathrm{~Hz}, 2 \mathrm{H}), 1.93(\mathrm{~m}, 1 \mathrm{H}), 0.89(\mathrm{~d}, J=6.6 \mathrm{~Hz}, 6 \mathrm{H})$;

PIVP ${ }^{1} \mathrm{H}-\mathrm{NMR}$ (DMSO- $\left.d_{6}, 400 \mathrm{MHz}\right), \delta 5.79(\mathrm{~s}, 2 \mathrm{H})$, $2.86(\mathrm{~d}, J=6.8 \mathrm{~Hz}, 2 \mathrm{H}), 2.13(\mathrm{~m}, 1 \mathrm{H}), 0.91(\mathrm{~d}, J=6.7 \mathrm{~Hz}$, $6 \mathrm{H})$.

\section{Results}

\section{Engineered synthesis of isovaleryl-CoA in E. coli}

First, we generated isovaleryl-CoA in E. coli cells employing a pathway from $M$. xanthus via hydroxy3-methylglutaryl CoA (HMG-CoA), a key intermediate of mevalonate pathway in the biosynthesis of isoprenoids [24]. Short branched-chain acyl-CoAs are usually derived from the branched-chain amino acids as a mixture via transamination and subsequent oxidative decarboxylation catalyzed by the branched-chain $\alpha$-keto acid dehydrogenase complex (BCDH) [26]. In current work, acetyl-CoA is converted into HMG-CoA as in the mevalonate pathway (Fig. 2a). Enzymes ERG10 (acetyl-CoA acetyltransferase) and ERG13 (HMG-CoA synthase) from S. cerevisiae were used for efficient biosynthesis of HMG-CoA, as these two enzymes yielded enhanced levels of mevalonate in E. coli [27-29]. The three enzymes from M. xanthus, including LiuC (HMGCoA dehydratase), AibA/B (3-methylglutaconyl-CoA (MG-CoA) decarboxylase) and AibC (3,3-dimethylacrylyl CoA (DMA-CoA) reductase), were used to transform HMG-CoA into isovaleryl-CoA consecutively via MGCoA and DMA-CoA in the above E. coli strain (Fig. 2a). Acyl-CoAs were extracted from the engineered $E$. coli strain APG-IV harboring pET-A and pCDF-E and analyzed by HPLC-MS with strain APG-0 harboring pETDuet-1 and pCDFDuet-1 as the negative control (Fig. 3). This procedure confirmed the production of isovalerylCoA by comparing the retention time $\left(\mathrm{t}_{R}=24.5 \mathrm{~min}\right)$ and the molecular ion $\left[(\mathrm{M}+\mathrm{H})^{+}=852.1802\right]$ with those of the isovaleryl-CoA standard (Fig. 3b, c). The titer of isovaleryl-CoA from this new pathway was $1421.8 \pm 129.2 \mathrm{nmol} / \mathrm{g}$ wet weight, about sixteen times higher than the titer of our previous engineered $E$. coli strain using the $\mathrm{BCDH}$ complex under the same fermentation and detection conditions (Fig. 3d) [26].

\section{Engineered synthesis of PIVP and HIBP in E. coli carrying} HIVPS $^{\text {syn }}$ and optimization of fermentation conditions The HlVPS $S^{\text {syn }}$ from hops was synthesized and introduced into the isovaleryl-CoA producing $E$. coli strain, generating the recombinant strain APG-1. After fermentation, the broth supernatants and cell pellets were analyzed by HPLC-MS. As shown in the chromatogram, the recombinant strain APG-1 produced two new compounds (Fig. 4c, d), compared with the control strain APG-0 (Fig. 4a, b). The first compound with a $\mathrm{t}_{R}$ of 35.6 min was initially identified as HIBP by LC-MS (Additional file 1: Figure S2a, c; $[\mathrm{M}+\mathrm{H}]^{+}=169.0763$ ). In the previous enzymatic studies, the production of HIBP was confirmed only by LC-MS analysis probably due to no enough products for NMR analysis [3, 23]. In this work, about $74.9 \mathrm{mg}$ HIBP was isolated from $2 \mathrm{~L}$ scale culture, and we were able to confirm the structure by $1 \mathrm{D}-\mathrm{NMR}$ spectroscopy analysis (Additional file 1 : Figure S2a, c). The second product with a $\mathrm{t}_{R}$ of $39.7 \mathrm{~min}$ was identified as PIVP (Additional file 1: Figure S2b, $\left.[\mathrm{M}+\mathrm{H}]^{+}=211.0925\right)$. About $6.5 \mathrm{mg} \mathrm{HIBP}$ was isolated from $2 \mathrm{~L}$ culture, and the PIVP structure was further confirmed by ${ }^{1} \mathrm{H}$-NMR spectroscopy analysis (Additional file 1: Figure S2d). The localization of the two products was also tested. PIVP mainly accumulated in the cell pellets (Fig. 4c), while HIBP was distributed in the fermentation broth as well as the cell pellets (Fig. 4c, d).

We further optimized the production of PIVP and HIBP by varying fermentation conditions for the recombinant strain APG-1 such as temperature and IPTG concentration at the first stage. The recombinant strain APG-1 was incubated in TB medium for $16 \mathrm{~h}$ at varying temperature $\left(16,23\right.$ or $\left.30^{\circ} \mathrm{C}\right)$ with a fixed IPTG concentration of $0.1 \mathrm{mM}$. The cell pellets were harvested and resuspended in modified M9 medium and incubated at $30{ }^{\circ} \mathrm{C}$ for $36 \mathrm{~h}$. Combining both the fermentation broth and cell pellets, the titerss of PIVP and HIBP exhibited best at $23{ }^{\circ} \mathrm{C}$ (Fig. 5a). After that, different concentrations of IPTG, 0.01, 0.05, 0.1, 0.2 and $0.4 \mathrm{mM}$ were used to induce protein expression at $23^{\circ} \mathrm{C}$. The titers of PIVP and HIBP were highest when the concentration of IPTG was $0.05 \mathrm{mM}$ as shown in Fig. 5b. Furthermore, we monitored titers of PIVP and HIBP produced by APG-1 cultivated in modified M9 medium at different fermentation time. The results are summarized in Fig. $5 \mathrm{c}$. The titers of HIBP and PIVP reached the highest in $24 \mathrm{~h}$, which were $52.0 \pm 7.9$ and $6.4 \pm 0.6 \mathrm{mg} / \mathrm{L}$, respectively. Under these concentrations, no inhibition on cell growth by the compounds HIBP and PIVP was observed.

\section{Biosynthesis of HIBP and PIVP in E. coli using CHSs}

In addition to HIVPS, a CHS from strawberry ( $F$. vesca), FvCHS2-1 played a role as a bifunctional CHS/VPS 

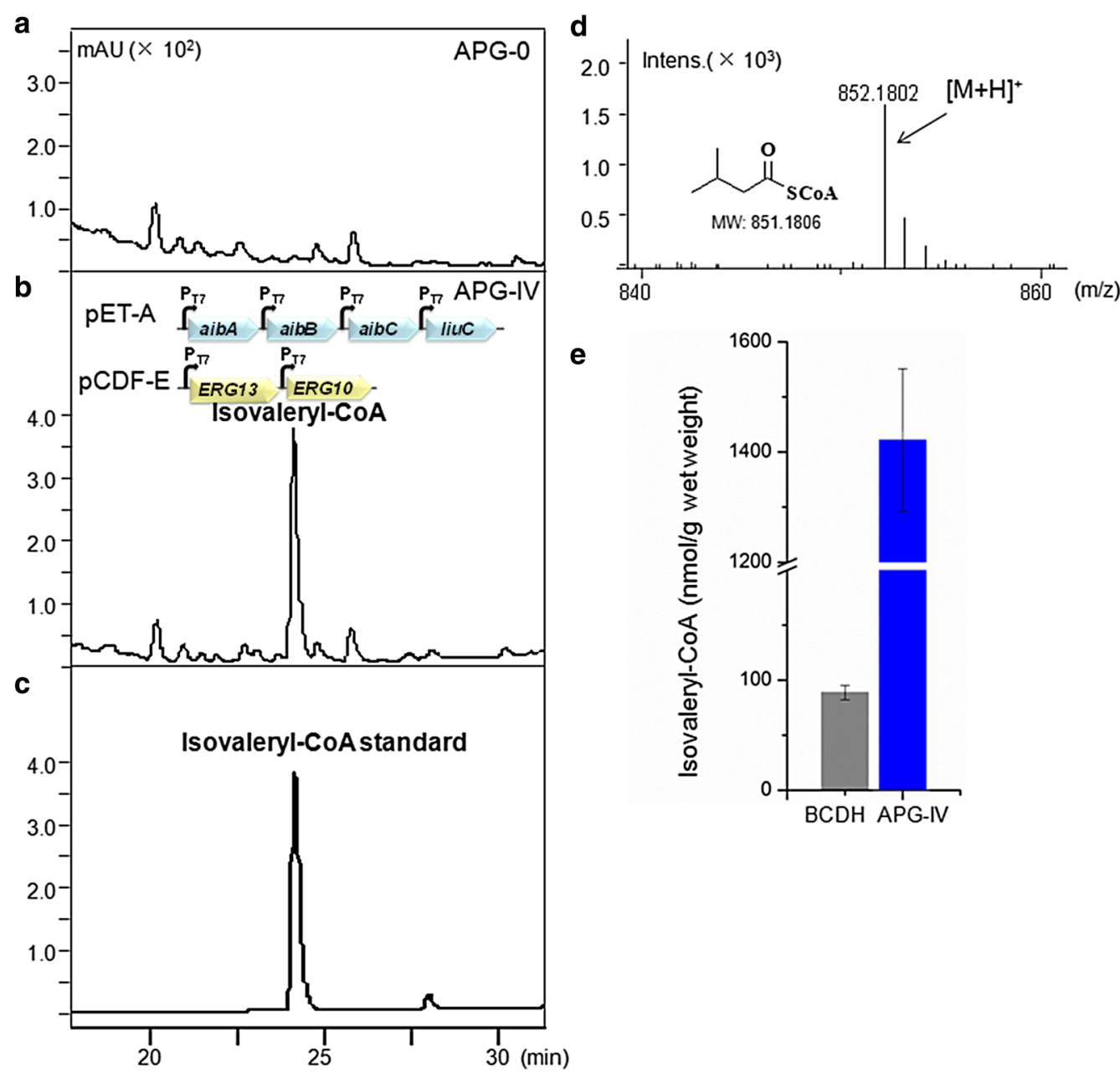

Fig. 3 Engineered synthesis of isovaleryl-CoA by E. coli strains. a HPLC analysis of chemical profiles produced by E. coli strain APG-0 harboring empty vectors. b HPLC analysis of isovaleryl-CoA produced by E. coli strain APG-IV carrying pET-A and pCDF-E. c The isovaleryl-CoA standard. d MS chromatogram showing the synthesis of isovaleryl-CoA. e Titers of isovaleryl-CoA produced by the recombinant strain APG-IV and our previous E. coli expressing the BCDH complex [26]

enzyme [3]. We performed codon optimization for the FvCHS2-1 gene, synthesized and introduced the $F v C H S 2-1^{\text {syn }}$ into the isovaleryl-CoA producing E. coli strain, generating APG-2 and fermented under the optimized conditions. The protein expression was induced at $23^{\circ} \mathrm{C}$ for $16 \mathrm{~h}$ with $0.05 \mathrm{mM}$ IPTG, and then the fermentation was conducted in modified M9 medium at $30{ }^{\circ} \mathrm{C}$ for $24 \mathrm{~h}$. HPLC analysis showed that the strain APG-2 also produced HIBP and PIVP, although the titer of PIVP was much lower than that carrying $H l V P S^{\text {syn }}$, only $1.4 \pm 0.2 \mathrm{mg} / \mathrm{L}$ (Fig. 4e, f, j). However, the titer of HIBP reached $53.3 \pm 9.0 \mathrm{mg} / \mathrm{L}$, which was similar to that of the strain harboring $H l V P S^{\text {syn }}$ (Fig. 4i).

VPS was involved in the formation of PIVP in the biosynthesis of humulone and could also produce PIPB using isobutyryl-CoA as the starter unit [21-23]. PIBP is the core structure of hyperforin, an antidepressant extracted from Hypericum species. The enzyme catalyzing formation of PIBP is still unknown. It was assumed that there may be a VPS-like or dual functional CHS/VPS enzyme in the Hypericum species responsible for the formation of PIBP, and more than likely, this enzyme could also use isovaleryl-CoA as the starter molecular to form PIVP and HIBP. Based on the reported type III PKSs gene sequences of the Hypericum species [30] in the NCBI database, several candidate genes were amplified from the callus of $H$. perforatum by homology cloning. Among these genes, a suspected CHS gene showing $96.2 \%$ similarity with FvCHS2-1 at the amino acid level was designated as HpCHS (GenBank: KU180217), and introduced into the above isovaleryl-CoA producer. The recombinant E. coli strain APG-3 harboring the isovaleryl-CoA 

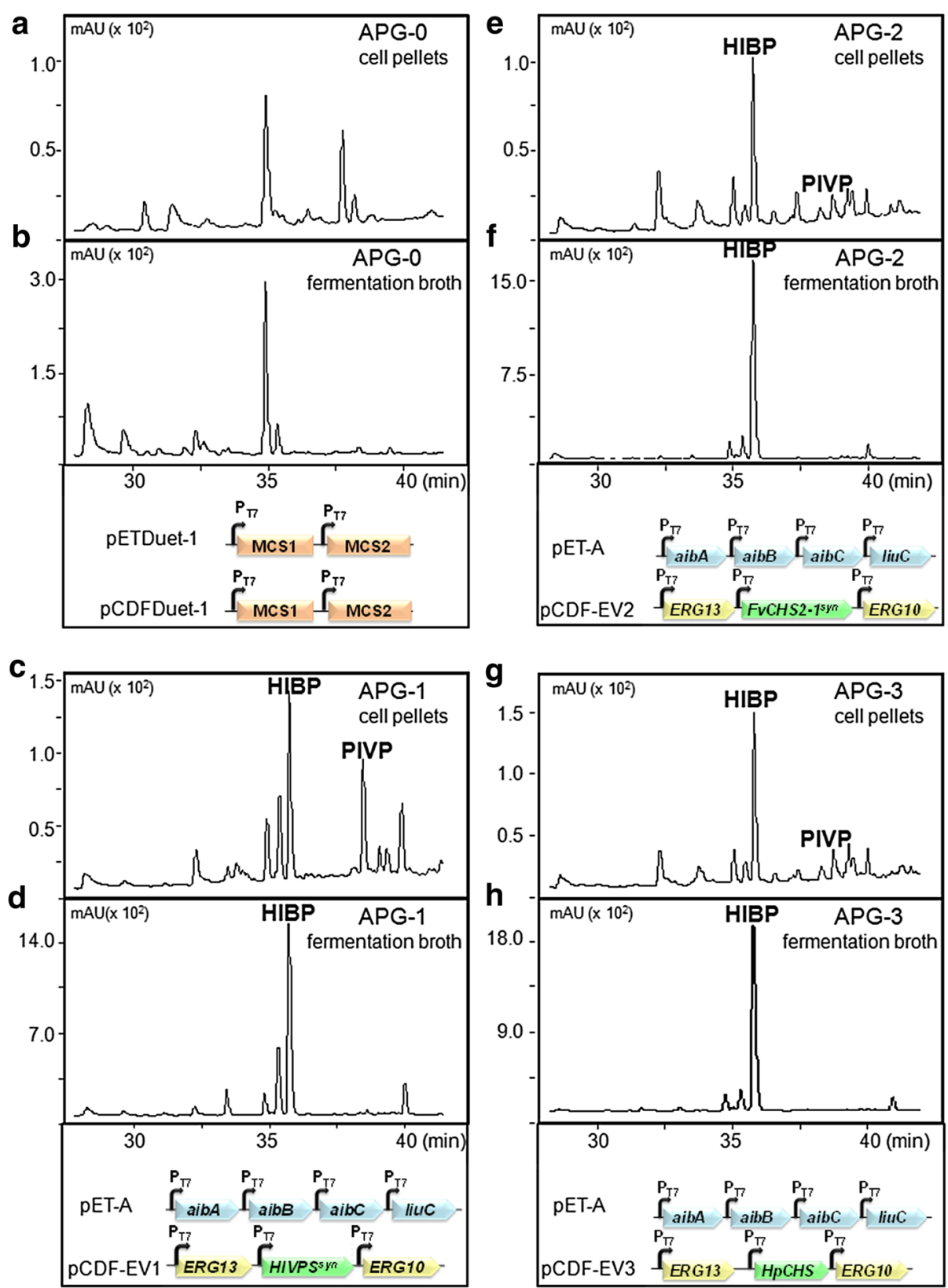

i
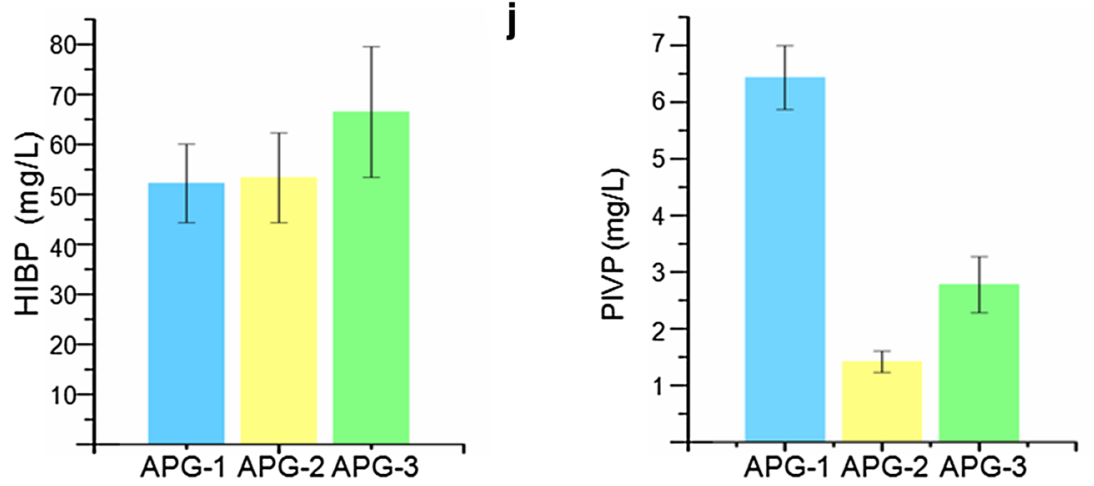
(See figure on previous page.)

Fig. 4 Engineered synthesis of 4-hydroxy-6-isovaleryl-2-pyrone (HIBP) and phlorisovalerophenone (PIVP) by E. coli strains. a, b Strain APG-0 harboring empty vectors as negative control. c, d Strain APG-1 harboring plasmids PET-A and pCDF-EV1. e, f Strain APG-2 harboring plasmids pET-A and pCDF-EV2. $\mathbf{g}$, h Strain APG-3 harboring plasmids pET-A and pCDF-EV3. a, c, e, $\mathbf{g}$ HPLC analysis of metabolites extracted from cell pellets.

$\mathbf{b}, \mathbf{d}, \mathbf{f}, \mathbf{h} \mathrm{HPLC}$ analysis of metabolites extracted from the fermentation broth. $\mathbf{i}$ The titers of HIBP produced by the strains APG-1, APG-2 and APG-3. $\mathbf{j}$ The titers of PIVP produced by the strains APG-1, APG-2 and APG-3

pathway genes and $H p C H S$ was fermented as described above. The HIBP titer produced by this strain reached $66.5 \pm 13.1 \mathrm{mg} / \mathrm{L}$, which was the highest among all the three producers (Fig. $4 \mathrm{~g}-\mathrm{i}$ ). The PIVP titer in APG-3 was $2.8 \pm 0.5 \mathrm{mg} / \mathrm{L}$, which was lower than that of the APG-1 harboring HlVPS ${ }^{s y n}$ (Fig. 4g, h, j).

\section{Discussion}

Short branched-chain acyl-CoAs are important building blocks for a large number of valuable compounds [5, 24, 31]. However, native E. coli metabolism does not produce these short branched-chain acyl-CoAs, which hampers the heterologous production of those economically important chemicals in this host. Previously engineered short branched-chain acyl-CoA producing E. coli strains usually biosynthesized isobutyryl-CoA, isovaleryl-CoA and 2-methyl-butyryl-CoA as a mixture derived from the metabolic pathways of branched-chain amino acids catalyzed by the BCDH complex [26, 32, 33]. In this study, we successfully demonstrated the feasibility of synthesizing isovaleryl-CoA in E. coli via HMG-CoA (Fig. 2a) recruiting five enzymes from yeast and myxobacteria [24]. Isovaleryl-CoA was the only product in this pathway which makes the engineered $E$. coli strain more suitable for producing compounds such as humulone and lupulone with isovaleryl-CoA as the building block. In the BCDH dependent pathway, isovaleryl-CoA and isobutyryl-CoA are derived from $\alpha$-ketoisocaproate or $\alpha$-ketoisovalerate directly, and $\alpha$-ketoisovalerate is an intermediate for the formation of $\alpha$-ketoisocaproate. Thus the formation of isobutyryl-CoA competes with isovaleryl-CoA in the pathway (Fig. 2a). Meanwhile short branched-chain keto acids would also be transformed into corresponding amino acids such as leucine, isoleucine and valine beyond acyl-CoAs (Fig. 2a). HMG-CoA formation with ERG10 and ERG13 derived from acetylCoA has been well established in the mevalonate pathway (Fig. 2a) in E. coli [27-29]. By recruiting LiuC, AibA/B and AibC, the HMG-CoA was further uniquely diverted into the formation of isovaleryl-CoA via MG-CoA and DMA-CoA. This engineered strain represents a novel "factory" for biosynthesis of a series of valuable chemicals with isovaleryl-CoA as the building block, including fatty acids, biofuels and natural products [26, 32, 33].

Introduction of VPS from hops, CHSs from strawberry or $H$. perforatum to the above isovaleryl-CoA producing E. coli strain resulted in the biosynthesis of PIVP and HIBP. PIVP was detected in hop extracts [34], and is the key intermediate of humulone, which contribute the characteristic bitter flavor of beer and display multiple pharmacological effects $[4,31]$. The work described here may pave the way for synthesis of humulone and other derivatives. HIBP has not been isolated from any natural resources [11-13]. As an analogue of TAL, HIBP could be used as a potential platform chemical to produce various chemical intermediates and end products with short
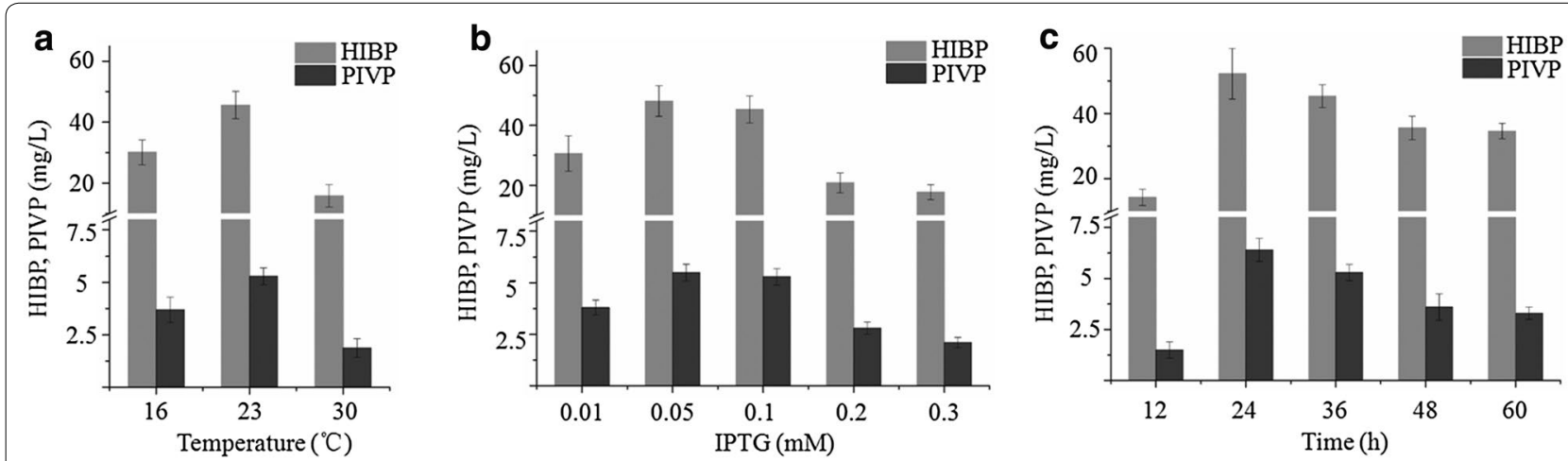

Fig. 5 Optimization of fermentation conditions. a The titers of PIVP and HIBP by strain APG-1 at different protein induction temperature. $\mathbf{b}$ The titers of PIVP and HIBP by strain APG-1 at different IPTG concentration. c The titers of PIVP and HIBP by strain APG-1 at different fermentation time 
branched-chains (Additional file 1: Figure S1a, b) [11-13, 35]. More experiments are needed to test whether HIBP itself has any antibacterial or fungicidal activity or not.

In this study, the VPS from hops was the best enzyme for PIVP formation. The newly cloned CHS from H. perforatum was the most appropriate enzyme for the synthesis of HIBP. The ratios of HIBP and PIVP were calculated based on the titers produced by strains APG-1 (52.0 over $6.4 \mathrm{mg} / \mathrm{L})$, APG-2 (53.3 over $1.4 \mathrm{mg} / \mathrm{L})$ and APG-3 (66.5 over $2.8 \mathrm{mg} / \mathrm{L}$ ), which were about $8: 1,37: 1$ and $24: 1$, respectively. In the in vitro enzyme activity analysis of HIVPS and FvCHS, PIVP was the major product, instead of HIBP [3, 22]. It was reported that the PIVP and HIBP content was affected by the relative ratio of isovaleryl$\mathrm{CoA} /$ malonyl-CoA in the in vitro experiment [3]. In our recombinant $E$. coli, the production of isovaleryl-CoA and malonyl-CoA both derived from acetyl-CoA, and there may be a dynamic balance between the concentration of isovaleryl-CoA and malonyl-CoA in vivo (Fig. 2a), which may affect the ratio of HIBP and PIVP.

Amino acids sequence alignment of HIVPS, FvCHS2-1 and $\mathrm{HpCHS}$ showed high similarity (Additional file 1: Figure S4). The three essential catalytic amino acids CysHis-Asn, the active site loop of CHS enzymes GFGPG, and two Phe residues, important in determining the substrate specificity of CHS are all well conserved in these three proteins (Additional file 1: Figure S4). However, ratios between PIVP and HIBP produced by these three enzymes are quite different (Fig. 4). Several protein engineering studies have elucidated the basis of starter molecule selectivity and the control of polyketide length of type III PKSs. For example, Jez and colleagues reported that a triple mutant (T197L/G256L/S338I) of CHS, generated an enzyme that was functionally identical to 2-PS, catalyzing the synthesis of the TAL from an acetyl-CoA starter molecule and two malonyl-CoAs [36]. In 2005, Abe and co-workers reported that a point mutation (M207G) in a pentaketide chromone synthase (PCS) expanded the volume of the catalytic cavity, to convert PCS into octaketide synthase [37]. Thus, modeling analyses and site-directed mutagenesis experiments are needed to improve the product specificity and increase the synthesis of PIVP or HIBP.

Directed evolution may be also used to increase the activity of HIVPS, FvCHS2-1 or HpCHS. Using directed evolution, Rao and colleagues obtained a series of thermostable PhlD mutants with improved phloroglucinol productivity [19]. Recently, Tang and colleagues engineered the $E$. coli regulatory protein AraC to activate gene expression in response to TAL, developed an endogenous TAL reporter system in $E$. coli. Using this system, they conducted in vivo directed evolution of 2-PS in $E$. coli, and obtained a 2-PS variant conferring $\sim 20$-fold higher TAL production [6]. Similar reporter systems may also be constructed in our recombinant E. coli to facilitate high throughput screening of HIVPS, FvCHS2-1 or $\mathrm{HpCHS}$ for improved productivity of HIBP or PIVP. At the same time, precursor supply including isovaleryl-CoA and malonyl-CoA may be improved for the production of HIBP and PIVP. The availability of intracellular malonylCoA may be enhanced by over-expressing the acetyl-CoA carboxylase (Acc) and acetyl-CoA synthase (Acs) genes and deletion of competing pathways, which was applied in improving phloroglucinol productivity by engineered E. coli strain $[19,38]$. In the case of HIBP and PIVP, as both substrates isovaleryl-CoA and malonyl-CoA were derived from acetyl-CoA, there may be a dynamic balance between these two substrates and may need more elaborate regulation.

\section{Conclusions}

In this work, we firstly constructed a biosynthetic pathway of isovaleryl-CoA in $E$. coli by recruiting a route via hydroxy-3-methylglutaryl $\mathrm{CoA}$, and then investigated the potential of bio-renewable production of PIVP and HIBP with E. coli as the host using type III PKSs. Even though still in infancy, the work described here may pave the way for microbial synthesis of not only acylphloroglucinols derivatives, but also "unnatural" TAL analogues.

\section{Additional file}

Additional file 1: Table S1. The main primers used in this study. Table S2. DNA sequences of synthesized genes. Figure S1. TAL was demonstrated to be a platform chemical and HIBP may also be used as a potential platform chemical. Figure S2. Mass spectrum and ${ }^{1} \mathrm{H}-\mathrm{NMR}$ analysis of HIBP and PIVP. Figure S3. Strain APG-IV and the E. coli strain harboring pET28a-HIVPS were also used as negative controls for the production of HIBP/PIVP. Figure S4. Comparison of the deduced amino acid sequences of HpCHS, FVCHS2-1 and HIVPS.

\section{Abbreviations}

2-PS: 2-pyrone synthase; BCDH: branched-chain a-keto acid dehydrogenase complex; CHS: chalcone synthase; DMA-CoA: 3,3-dimethylacrylyl CoA; HIBP: 4-hydroxy-6-isobutyl-2-pyrone; HIPP: 4-hydroxy-6-isopropyl-2-pyrone; HMGCoA: hydroxy-3-methylglutaryl COA; IPTG: isopropyl- $\beta$ - $d$-thiogalactoside; MG-COA: 3-methylglutaconyl-COA; PCS: pentaketide chromone synthase; PIBP: phlorisobutyrophenone; PIVP: phlorisovalerophenone; PKSs: polyketide synthases; TAL: triacetic acid lactone; VPS: valerophenone synthase.

\section{Authors' contributions}

$T L, H B, W Z, Y Z, Y B$ and $Y M$ designed the project. $W Z, H B, Y Z$ and $Y B$ performed the experiments. WZ, HB, $Y Z$ and $T L$ analyzed the data. $T L, H B, W Z$ and $Y Z$ wrote the manuscript. All authors read and approved the final manuscript.

\section{Author details \\ ${ }^{1}$ Tianjin Institute of Industrial Biotechnology, Chinese Academy of Sciences, Tianjin 300308, China. ${ }^{2}$ Key Laboratory of Systems Microbial Biotechnology, Chinese Academy of Sciences, Tianjin 300308, China. ${ }^{3}$ University of Chinese Academy of Sciences, Beijing, China.}

\section{Acknowledgements}

None. 


\section{Competing interests}

The authors declare that they have no competing interests.

\section{Availability of data and materials}

Codon-optimized sequences generated in this project are listed in Additional file 1: Table S2. The HpCHS sequence was submitted into Genbank (http:// www.ncbi.nlm.nih.gov/) and the accession number was indicated in the "Methods" section.

\section{Ethics approval and consent to participate}

This manuscript dose not report data collected from humans or animals.

\section{Funding}

This work was supported by grants from the 973 Program of China (2012CB721100), the National Natural Science Foundation of China (31300040 and 21302214).

\section{Received: 20 June 2016 Accepted: 22 August 2016}

Published online: 30 August 2016

\section{References}

1. Bohr G, Gerhauser C, Knauft J, Zapp J, Becker H. Anti-inflammatory acylphloroglucinol derivatives from Hops (Humulus lupulus). J Nat Prod. 2005:68:1545-8.

2. Crispin MC, Hur M, Park T, Kim YH, Wurtele ES. Identification and biosynthesis of acylphloroglucinols in Hypericum gentianoides. Physiol Plant. 2013;148:354-70.

3. Song C, Ring L, Hoffmann T, Huang FC, Slovin JP, Schwab W. Acylphloroglucinol biosynthesis in strawberry fruit. Plant Physiol. 2015;15:00794.

4. Clark SM, Vaitheeswaran V, Ambrose SJ, Purves RW, Page JE. Transcriptome analysis of bitter acid biosynthesis and precursor pathways in hop (Humulus lupulus). BMC Plant Biol. 2013;13:12

5. Barnes J, Anderson LA, Phillipson JD. St John's wort (Hypericum perforatum L.): a review of its chemistry, pharmacology and clinical properties. J Pharm Pharmacol. 2001:53:583-600.

6. Tang SY, Qian S, Akinterinwa O, Frei CS, Gredell JA, Cirino PC. Screening for enhanced triacetic acid lactone production by recombinant Escherichia coli expressing a designed triacetic acid lactone reporter. J Am Chem Soc. 2013;135:10099-103.

7. Eckermann S, Schroder G, Schmidt J, Strack D, Edrada RA, Helariutta Y, Elomaa P, Kotilainen M, Kilpelainen I, Proksch P, et al. New pathway to polyketides in plants. Nature. 1998:396:387-90.

8. Igarashi Y, Ogawa M, Sato Y, Saito N, Yoshida R, Kunoh H, Onaka H, Furumai T. Fistupyrone, a novel inhibitor of the infection of Chinese cabbage by Alternaria brassicicola, from Streptomyces sp. TP-A0569. J Antibiot (Tokyo). 2000;53:1117-22

9. Petersen F, Zahner H, Metzger JW, Freund S, Hummel RP. Germicidin, an autoregulative germination inhibitor of Streptomyces viridochromogenes NRRL B-1551. J Antibiot (Tokyo). 1993:46:1126-38.

10. Aoki Y, Matsumoto D, Kawaide H, Natsume M. Physiological role of germicidins in spore germination and hyphal elongation in Streptomyces coelicolor A3(2). J Antibiot (Tokyo). 2011;64:607-11.

11. Chia M, Schwartz TJ, Shanks BH, Dumesic JA. Triacetic acid lactone as a potential biorenewable platform chemical. Green Chem. 2012;14:1850-3.

12. Dumesic JA, Chia M. Production of 2,4-diones from 4-hydroxy6-substituted-2-pyrones. 2013; United State Patent Application, No. 20120283477A1.

13. Schwartz TJ, O'Neill BJ, Shanks BH, Dumesic JA. Chemlnform abstract: bridging the chemical and biological catalysis gap: challenges and outlooks for producing sustainable chemicals. Chemlnform. 2014;45:2060-9.

14. Mora-Pale M, Sanchez-Rodriguez SP, Linhardt RJ, Dordick JS, Koffas MA. Metabolic engineering and in vitro biosynthesis of phytochemicals and non-natural analogues. Plant Sci. 2013;210:10-24.

15. Trantas EA, Koffas MA, Xu P, Ververidis F. When plants produce not enough or at all: metabolic engineering of flavonoids in microbial hosts. Front Plant Sci. 2015:6:7.
16. Xie D, Shao Z, Achkar J, Zha W, Frost JW, Zhao H. Microbial synthesis of triacetic acid lactone. Biotechnol Bioeng. 2006;93:727-36.

17. Achkar J, Xian M, Zhao H, Frost JW. Biosynthesis of phloroglucinol. J Am Chem Soc. 2005;127:5332-3.

18. Cardenas J, Da Silva NA. Metabolic engineering of Saccharomyces cerevisiae for the production of triacetic acid lactone. Metab Eng. 2014:25:194-203.

19. Rao G, Lee J-K, Zhao H. Directed evolution of phloroglucinol synthase PhID with increased stability for phloroglucinol production. Appl Microbiol Biotechnol. 2013;97:5861-7.

20. Adam P, Arigoni D, Bacher A, Eisenreich W. Biosynthesis of hyperforin in Hypericum perforatum. J Med Chem. 2002;45:4786-93.

21. Novák P, Matoušek J, Bř́za J. Valerophenone synthase-like chalcone synthase homologues in Humulus lupulus. Biol Plant. 2003;46:375-81.

22. Okada Y, Ito K. Cloning and analysis of valerophenone synthase gene expressed specifically in lupulin gland of hop (Humulus lupulus L.). Biosci Biotechnol Biochem. 2001;65:150-5.

23. Paniego NB, Zuurbier KW, Fung SY, van der Heijden R, Scheffer JJ, Verpoorte R. Phlorisovalerophenone synthase, a novel polyketide synthase from hop (Humulus lupulus L.) cones. Eur J Biochem. 1999:262:612-6.

24. Li Y, Luxenburger E, Muller R. An alternative isovaleryl CoA biosynthetic pathway involving a previously unknown 3-methylglutaconyl CoA decarboxylase. Angew Chem Int Ed Engl. 2013;52:1304-8.

25. Engler C, Marillonnet S. Golden Gate cloning. Methods Mol Biol. 2014;1116:119-31.

26. Bi H, Bai Y, Cai T, Zhuang Y, Liang X, Zhang X, Liu T, Ma Y. Engineered short branched-chain acyl-CoA synthesis in E. coli and acylation of chloramphenicol to branched-chain derivatives. Appl Microbiol Biotechnol. 2013:97:10339-48.

27. Martin VJ, Pitera DJ, Withers ST, Newman JD, Keasling JD. Engineering a mevalonate pathway in Escherichia coli for production of terpenoids. Nat Biotechnol. 2003;21:796-802.

28. Steen EJ, Chan R, Prasad N, Myers S, Petzold CJ, Redding A, Ouellet M, Keasling JD. Metabolic engineering of Saccharomyces cerevisiae for the production of n-butanol. Microb Cell Fact. 2008;7:36.

29. Zhu F, Zhong X, Hu M, Lu L, Deng Z, Liu T. In vitro reconstitution of mevalonate pathway and targeted engineering of farnesene overproduction in Escherichia coli. Biotechnol Bioeng. 2014;111:1396-405.

30. Karppinen K, Hokkanen J, Mattila S, Neubauer P, Hohtola A. Octaketide-producing type III polyketide synthase from Hypericum perforatum is expressed in dark glands accumulating hypericins. FEBS J. 2008:275:4329-42.

31. Van Cleemput M, Cattoor K, De Bosscher K, Haegeman G, De Keukeleire D, Heyerick A. Hop (Humulus lupulus)-derived bitter acids as multipotent bioactive compounds. J Nat Prod. 2009;72:1220-30.

32. Atsumi S, Hanai T, Liao JC. Non-fermentative pathways for synthesis of branched-chain higher alcohols as biofuels. Nature. 2008:451:86-9.

33. Howard TP, Middelhaufe S, Moore K, Edner C, Kolak DM, Taylor GN, Parker DA, Lee R, Smirnoff N, Aves SJ, Love J. Synthesis of customized petroleumreplica fuel molecules by targeted modification of free fatty acid pools in Escherichia coli. Proc Natl Acad Sci USA. 2013:110:7636-41.

34. Zuurbier KWM, Fung S-Y, Scheffer JJC, Verpoorte R. Formation of aromatic intermediates in the biosynthesis of bitter acids in Humulus lupulus. Phytochemistry. 1995;38:77-82.

35. Burns MJ, Ronson TO, Taylor RJ, Fairlamb IJ. 4-Hydroxy-6-alkyl-2-pyrones as nucleophilic coupling partners in Mitsunobu reactions and oxa-Michael additions. Beilstein J Org Chem. 2014;10:1159-65.

36. Jez JM, Austin MB, Ferrer J, Bowman ME, Schroder J, Noel JP. Structural control of polyketide formation in plant-specific polyketide synthases. Chem Biol. 2000:7:919-30.

37. Abe I, Utsumi Y, Oguro S, Morita H, Sano Y, Noguchi H. A plant type III polyketide synthase that produces pentaketide chromone. J Am Chem Soc. 2005; 127:1362-3

38. Zha WJ, Rubin-Pitel SB, Shao ZY, Zhao HM. Improving cellular malonylCoA level in Escherichia coli via metabolic engineering. Metab Eng. 2009;11:192-8. 